\title{
Computation and Storage of the Bianisotropic Scalar Green's Function and its Derivatives
}

\author{
Ignace Bogaert, Member, IEEE
}

\begin{abstract}
A set of algorithms is proposed for the accurate and efficient computation and storage of the bianisotropic scalar Green's function. The computation is based on an expansion of the Green's function into Chebyshev polynomials. The analytical properties of these polynomials are exploited to allow the accurate computation of the derivatives of the Green's function as well as the Green's function itself. For lossy materials, the proposed computation strategy is provably robust. In addition, a multilevel storage scheme with a favorable complexity, based on the Chebyshev polynomial expansion, is proposed for the storage of the expansion coefficients. Numerical results showcase the accuracy and computational complexity of the proposed algorithms.
\end{abstract}

Index Terms-Bianisotropic Materials, Scalar Green's Function, Computation, Storage

\section{INTRODUCTION}

I NTEGRAL equations are among the most successful formulations for the solution of electromagnetic scattering problems. They combine the exact incorporation of the radiation condition with the key advantage of not having to discretize the volume of piecewise homogeneous scatterers. In principle, integral equations can be used just as well for bianisotropic media as for the more common isotropic media. However, the literature contains few attempts to do this (an example is [1], [2]). This can probably be traced to the fact that the Green's dyadics are generally not available in closed form, despite the impressive progress made in the past decades [3]-[5]. This simple fact complicates the usual steps in the Method of Moments (MoM [6]), e.g. the computation of the Green's dyadics, the computation of the matrix elements and the evaluation of the scattered fields.

The work in this paper is focussed on the first step required for the successful application of the MoM to bianisotropic media: the computation of the bianisotropic scalar Green's function (BSGF) and its derivatives. If this computation can be accurately performed, it opens the way to the computation of the Green's dyadics and, ultimately, of the MoM matrix elements and scattered fields. Since the computation of the BSGF is a computationally costly operation, it also makes sense to develop a storage scheme that allows the pre-computation of the BSGF, such that the repeated evaluation of the BSGF can be done not only accurately, but also efficiently.

The proposed storage scheme is based on an expansion of the BSGF into Chebyshev polynomials [7], which is a special case of the expansion presented in [8]. The storage scheme derives a number of important advantages from this. First, the Chebyshev polynomials are very well-suited for

I. Bogaert is with the Department of Information Technology, Ghent University, Belgium, e-mail: Ignace.Bogaert@intec.UGent.be the approximation of functions, achieving high accuracy and stability with a nearly optimal number of terms [9]. Second, the properties of the Chebyshev polynomials allow some pertinent integrals to be computed analytically, resulting in Bessel functions. Because of the existence of software for the accurate computation of Bessel functions (for example a wellknown library by D. E. Amos [10]), even those that are much smaller than the machine precision, this allows for the accurate computation of very small Chebyshev expansion coefficients. A third advantage is that the occurrence of the Bessel functions can be leveraged to remove a removable singularity that otherwise could degrade or destroy the precision of the numerically computed BSGF.

It should be made clear from the start that the algorithms in this paper cannot be expected to work on every bianisotropic material imaginable. This would be pretty much impossible because of the sheer size of the parameter space (36 complex parameters) involved. Instead we will focus on the subclass of lossy bianisotropic materials, which allows the rigorous proof of many properties that are used. In practice, many bianisotropic materials have at least some loss, such that the techniques described here are widely applicable. Also note that the loss can be made arbitrarily small, such that many lossless materials can be handled in a good approximation.

The paper outline is as follows: first, the theory behind the BSGF is briefly reviewed, and the link with the computation of the Green's dyadics is made (Section II). Second, a number of theoretical results pertaining to lossy materials are proved (Section III). Third, the computation strategy for the BSGF expansion coefficients is presented (Section IV), followed by the treatment of a numerically pathological case (Section V). Fourth, the computation of the derivatives is discussed (Section VI). Then, a multilevel data structure is proposed for the storage of the BSGF with a favorable complexity (Section VII). Finally, numerical results are presented to showcase the accuracy of the proposed techniques (Section VIII). Throughout this paper, an $e^{j \omega t}$ time dependence is assumed and suppressed.

\section{The Bianisotropic Scalar Green's Function}

In this Section, the BSGF will be defined and its role in the computation of electric and magnetic fields explained. Much of this material can be found in the literature [11], [12], but we will briefly summarize the most important concepts here for further reference in the rest of this paper. The BSGF occurs in the study of the fields radiated by elementary dipole sources, 
which are electric and magnetic currents given by

$$
\begin{aligned}
\boldsymbol{j}(\boldsymbol{r}) & =\boldsymbol{j} \delta(\boldsymbol{r}), \\
\boldsymbol{m}(\boldsymbol{r}) & =\boldsymbol{m} \delta(\boldsymbol{r}) .
\end{aligned}
$$

Of course, the fields generated by such elementary dipoles are just the Green dyadics:

$$
\begin{aligned}
\boldsymbol{e}(\boldsymbol{r}) & =\overline{\bar{G}}_{e e}(\boldsymbol{r}) \cdot \boldsymbol{j}+\overline{\bar{G}}_{e m}(\boldsymbol{r}) \cdot \boldsymbol{m}, \\
\boldsymbol{h}(\boldsymbol{r}) & =\overline{\bar{G}}_{m e}(\boldsymbol{r}) \cdot \boldsymbol{j}+\overline{\bar{G}}_{m m}(\boldsymbol{r}) \cdot \boldsymbol{m} .
\end{aligned}
$$

The Green dyadics are computed using Maxwell's curl equations

$$
\begin{aligned}
\nabla \times \boldsymbol{h}(\boldsymbol{r})-j \omega \boldsymbol{d}(\boldsymbol{r}) & =\boldsymbol{j} \delta(\boldsymbol{r}), \\
-\nabla \times \boldsymbol{e}(\boldsymbol{r})-j \omega \boldsymbol{b}(\boldsymbol{r}) & =\boldsymbol{m} \delta(\boldsymbol{r}),
\end{aligned}
$$

and the constitutive equations for a general linear homogeneous bianisotropic medium

$$
\begin{aligned}
& \boldsymbol{d}(\boldsymbol{r})=\overline{\bar{\varepsilon}} \cdot \boldsymbol{e}(\boldsymbol{r})+\overline{\bar{\xi}} \cdot \boldsymbol{h}(\boldsymbol{r}), \\
& \boldsymbol{b}(\boldsymbol{r})=\overline{\bar{\zeta}} \cdot \boldsymbol{e}(\boldsymbol{r})+\overline{\bar{\mu}} \cdot \boldsymbol{h}(\boldsymbol{r}) .
\end{aligned}
$$

Throughout the rest of this paper, it will sometimes be useful to organize these constitutive equations into a single matrix equation

$$
\left[\begin{array}{l}
\boldsymbol{d}(\boldsymbol{r}) \\
\boldsymbol{b}(\boldsymbol{r})
\end{array}\right]=\underbrace{\left[\begin{array}{cc}
\overline{\bar{\varepsilon}} & \overline{\bar{\xi}} \\
\overline{\bar{\zeta}} & \overline{\bar{\mu}}
\end{array}\right]}_{\mathrm{M}} \cdot\left[\begin{array}{l}
\boldsymbol{e}(\boldsymbol{r}) \\
\boldsymbol{h}(\boldsymbol{r})
\end{array}\right]
$$

where the matrix containing the material dyadics will be denoted by $\mathrm{M}$ and called the material matrix. A medium is called anisotropic (instead of bianisotropic) when the magnetoelectric coupling tensors, i.e. $\overline{\bar{\xi}}$ and $\overline{\bar{\zeta}}$, are zero. In the isotropic case, all parameter tensors are scalars times the identity tensor. For the computation of the Green dyadics, the bianisotropic medium is assumed to extend to infinity in all directions. This allows us to perform a spatial Fourier transform of these equations, which replaces $\nabla$ with $j \boldsymbol{k}, \boldsymbol{e}(\boldsymbol{r})$ with $\hat{\boldsymbol{e}}(\boldsymbol{k})$ and $\boldsymbol{h}(\boldsymbol{r})$ with $\hat{\boldsymbol{h}}(\boldsymbol{k})$. The following concise matrix form can then be constructed:

$$
-j \omega \mathrm{P}\left(\frac{\boldsymbol{k}}{\omega}\right) \cdot\left[\begin{array}{l}
\hat{e}(\boldsymbol{k}) \\
\hat{h}(k)
\end{array}\right]=\left[\begin{array}{c}
\boldsymbol{j} \\
\boldsymbol{m}
\end{array}\right]
$$

with

$$
\mathrm{P}(s)=\left[\begin{array}{cc}
\overline{\bar{\varepsilon}} & \overline{\bar{\xi}}-s \times \mathbb{1} \\
\overline{\bar{\zeta}}+s \times \mathbb{1} & \overline{\bar{\mu}}
\end{array}\right]
$$

and

$$
s \times \mathbb{1}=\left[\begin{array}{ccc}
0 & -s_{z} & s_{y} \\
s_{z} & 0 & -s_{x} \\
-s_{y} & s_{x} & 0
\end{array}\right] .
$$

To get the fields generated by the dipole currents, the matrix $P$ needs to be inverted

$$
\left[\begin{array}{l}
\hat{e}(\boldsymbol{k}) \\
\hat{\boldsymbol{h}}(\boldsymbol{k})
\end{array}\right]=\frac{j}{\omega} \mathrm{P}^{-1}\left(\frac{\boldsymbol{k}}{\omega}\right) \cdot\left[\begin{array}{c}
\boldsymbol{j} \\
\boldsymbol{m}
\end{array}\right]
$$

and inverse Fourier-transformed, which yields

$$
\begin{aligned}
{\left[\begin{array}{cc}
\overline{\bar{G}}_{e e}(\boldsymbol{r}) & \overline{\bar{G}}_{e m}(\boldsymbol{r}) \\
\overline{\bar{G}}_{m e}(\boldsymbol{r}) & \overline{\bar{G}}_{m m}(\boldsymbol{r})
\end{array}\right] } & =\frac{j}{8 \pi^{3} \omega} \int_{\mathbb{R}^{3}} e^{j \boldsymbol{k} \cdot \boldsymbol{r}} \mathrm{P}^{-1}\left(\frac{\boldsymbol{k}}{\omega}\right) \mathrm{d} \boldsymbol{k}, \\
& =\frac{j \omega^{2}}{8 \pi^{3}} \int_{\mathbb{R}^{3}} e^{j \omega \boldsymbol{s} \cdot \boldsymbol{r}} \mathrm{P}^{-1}(\boldsymbol{s}) \mathrm{d} \boldsymbol{s} .
\end{aligned}
$$

Here, the integration over three dimensional $\boldsymbol{k}$-space was replaced by an integration over the so-called slowness $s=\frac{k}{\omega}$ [11]. It is possible to simplify this result further by writing the inverse of $\mathrm{P}$ as its adjugate divided by its determinant

$$
\begin{aligned}
\mathrm{P}^{-1}(\boldsymbol{s}) & =\frac{\mathrm{A}(\boldsymbol{k})}{D(\boldsymbol{s})}, \\
\mathrm{A}(\boldsymbol{s}) & =\operatorname{Adj}[\mathrm{P}(\boldsymbol{s})], \\
D(s) & =\operatorname{Det}[\mathrm{P}(s)] .
\end{aligned}
$$

The adjugate of a matrix is obtained by replacing every element with its cofactor and transposing the resulting matrix (see [13]). The determinant $D(s)$ is a polynomial of degree four in the components of $s$ and is usually called the Helmholtz determinant [11], [12]. Because the elements of $P$ are polynomials in the components of $s$, it follows that the elements of the adjugate matrix $\mathrm{A}(\boldsymbol{s})$ are also polynomials in the components of $s$. This means that the adjugate matrix can be brought outside of the integral, if $s$ is replaced with $-j / \omega \nabla$.

$$
\begin{aligned}
{\left[\begin{array}{cc}
\overline{\bar{G}}_{e e}(\boldsymbol{r}) & \overline{\bar{G}}_{e m}(\boldsymbol{r}) \\
\overline{\bar{G}}_{m e}(\boldsymbol{r}) & \overline{\bar{G}}_{m m}(\boldsymbol{r})
\end{array}\right] } & =\frac{j \omega^{2}}{8 \pi^{3}} \mathrm{~A}(-j / \omega \boldsymbol{\nabla}) \int_{\mathbb{R}^{3}} \frac{e^{j \omega \boldsymbol{s} \cdot \boldsymbol{r}}}{D(\boldsymbol{s})} \mathrm{d} \boldsymbol{s} \\
& =j \omega^{2} \mathrm{~A}(-j / \omega \boldsymbol{\nabla}) G(\omega \boldsymbol{r}) .
\end{aligned}
$$

where the frequency-independent bianisotropic scalar Green's function, defined by

$$
G(\boldsymbol{r})=\frac{1}{8 \pi^{3}} \int_{\mathbb{R}^{3}} \frac{e^{j \boldsymbol{s} \cdot \boldsymbol{r}}}{D(\boldsymbol{s})} \mathrm{d} \boldsymbol{s},
$$

was used.

Equation (15) shows that, if the BSGF can be computed, the Green's dyadics for the electric and magnetic fields can also be computed. However, there is an important technicality that should be borne in mind: the application of the operator A $\left(-j \frac{\boldsymbol{\nabla}}{\omega}\right)$ to the BSGF entails taking repeated derivatives. A lengthy calculation shows that the highest-order derivatives that occur are fourth-order derivatives. If an analytical expression is known for the BSGF, taking derivatives is not a problem. However, if the BSGF is computed numerically, then it inevitably contains some error compared to the exact solution. Each time a derivative is taken of such a numerically computed BSGF, the error is increased. Therefore, it can be hard to accurately evaluate the fourth-order derivatives occurring in (15).

\section{The Helmholtz Determinant in LOSSY MATERIALS}

The Helmholtz determinant (14), defined in the previous Section, is a polynomial of degree four in the vector components of the slowness $s$. However, due to the total freedom 
in the choice of the material, the Helmholtz determinant's 35 polynomial coefficients can be virtually anything. This fact makes finding algorithms (for the computation of the BSGF) that work for any material nigh impossible. However, as will be shown in this Section, the properties of the Helmholtz determinant become remarkably tractable if the assumption is made that the material is lossy.

Definition A bianisotropic material is called lossy if and only if

$$
\Im\left\{\boldsymbol{V}^{H} \cdot \mathrm{M} \cdot \boldsymbol{V}\right\}<0, \forall \boldsymbol{V} \in \mathbb{C}^{6} \backslash\{\mathbf{0}\},
$$

where the matrix $M$ is the material matrix containing the four material dyadics (see equation (5)).

From this definition, it is easily shown that the collection of all lossy materials constitutes a convex set (in 72-dimensional space):

Theorem 3.1: Consider two lossy materials, specified by their material dyadics $\mathrm{M}_{0}$ and $\mathrm{M}_{1}$

$$
\mathbf{M}_{0}=\left[\begin{array}{cc}
\overline{\bar{\varepsilon}}_{0} & \overline{\bar{\xi}}_{0} \\
\overline{\bar{\zeta}}_{0} & \overline{\bar{\mu}}_{0}
\end{array}\right], \mathbf{M}_{1}=\left[\begin{array}{cc}
\overline{\bar{\varepsilon}}_{1} & \overline{\bar{\xi}}_{1} \\
\bar{\zeta}_{1} & \overline{\bar{\mu}}_{1}
\end{array}\right] \text {. }
$$

Then the material with material matrix $\mathrm{M}_{\alpha}$ given by

$$
\mathrm{M}_{\alpha}=(1-\alpha) \mathrm{M}_{0}+\alpha \mathrm{M}_{1}
$$

is also lossy if $\alpha \in[0,1]$.

Proof: For $\alpha=0$ or 1 , the theorem is trivially true because of the assumptions of the theorem. For $\alpha \in] 0,1[$, start by writing

$$
\begin{aligned}
& \Im\left\{\boldsymbol{V}^{H} \cdot \mathrm{M}_{\alpha} \cdot \boldsymbol{V}\right\}=(1-\alpha)\left\{\boldsymbol{V}^{H} \cdot \mathrm{M}_{0} \cdot \boldsymbol{V}\right\} \\
&+\alpha \Im\left\{\boldsymbol{V}^{H} \cdot \mathrm{M}_{1} \cdot \boldsymbol{V}\right\} .
\end{aligned}
$$

Because the factors $(1-\alpha)$ and $\alpha$ are positive, and the fact that both $M_{0}$ and $M_{1}$ satisfy (17), this immediately shows that

$$
\Im\left\{\boldsymbol{V}^{H} \cdot \mathrm{M}_{\alpha} \cdot \boldsymbol{V}\right\}<0, \forall \boldsymbol{V} \in \mathbb{C}^{6} \backslash\{\mathbf{0}\},
$$

which proves the theorem.

Theorem 3.1 basically states that every material matrix on the straight line (in $\mathbb{C}^{36}$ ) between two lossy materials is also lossy. Hence the lossy materials form a convex set.

Another fundamental property of lossy materials is that, for real slowness $s$, the Helmholtz determinant $D(s)$ cannot become zero.

Theorem 3.2: If a material with material matrix $M$ is lossy, then

$$
D(s) \neq 0, \forall s \in \mathbb{R}^{3} .
$$

Proof: This proof will proceed by assuming that there exists a real zero $s_{0}$ of the Helmholtz determinant and showing that this results in a contradiction. If

$$
D\left(s_{0}\right)=0,
$$

then, by the definition of the Helmholtz determinant (14), there exists a complex vector $\boldsymbol{V}_{0} \in \mathbb{C}^{6}$ such that

$$
\left[\begin{array}{cc}
\overline{\bar{\varepsilon}} & \overline{\bar{\xi}}-\boldsymbol{s}_{0} \times \mathbb{1} \\
\overline{\bar{\zeta}}+\boldsymbol{s}_{0} \times \mathbb{1} & \overline{\bar{\mu}}
\end{array}\right] \cdot \boldsymbol{V}_{0}=\mathbf{0} .
$$

Taking the imaginary part of the inner product with $\boldsymbol{V}_{0}^{H}$ yields

$$
\Im\left\{\boldsymbol{V}_{0}^{H} \cdot\left[\begin{array}{cc}
\overline{\bar{\varepsilon}} & \overline{\bar{\xi}}-\boldsymbol{s}_{0} \times \mathbb{1} \\
\overline{\bar{\zeta}}+\boldsymbol{s}_{0} \times \mathbb{1} & \overline{\bar{\mu}}
\end{array}\right] \cdot \boldsymbol{V}_{0}\right\}=0 .
$$

However, if $s_{0}$ is real, then

$$
\Im\left\{\boldsymbol{V}_{0}^{H} \cdot\left[\begin{array}{cc}
0 & -\boldsymbol{s}_{0} \times \mathbb{1} \\
\boldsymbol{s}_{0} \times \mathbb{1} & 0
\end{array}\right] \cdot \boldsymbol{V}_{0}\right\}=0,
$$

regardless of what $\boldsymbol{V}_{0}$ may be, because the matrix in between the two vectors is hermitian. Therefore, (25) simplifies to

$$
\Im\left\{\boldsymbol{V}_{0}^{H} \cdot\left[\begin{array}{cc}
\overline{\bar{\varepsilon}} & \overline{\bar{\xi}} \\
\overline{\bar{\zeta}} & \overline{\bar{\mu}}
\end{array}\right] \cdot \boldsymbol{V}_{0}\right\}=0,
$$

which contradicts the criterium for lossyness (17).

Surprisingly, the fact that a material is lossy also guarantees that there are exactly four complex solutions for $\lambda$ to the equation

$$
D\left(\boldsymbol{s}_{a}+\lambda \boldsymbol{s}_{b}\right)=0 .
$$

When $s_{a}=\mathbf{0}$, this equation describes the problem of finding the plane wave solutions of Maxwell's equations, with propagation direction parallel to $s_{b}$. It is widely known that usually, bianisotropic materials admit four independent solutions. However, it is very well possible to propose theoretical materials for which the number of solutions is less than four. Since equation (28) is repeatedly solved in the proposed algorithm for the computation of the BSGF, a lot of pathological cases would be eliminated by knowing in advance that there will always be four solutions. Fortunately, this can be proved rigorously. The theorem statement is as follows:

Theorem 3.3: For all $\boldsymbol{s}_{a}, \boldsymbol{s}_{b} \in \mathbb{R}^{3} \backslash\{\mathbf{0}\}$, and $D(\boldsymbol{s})$ the Helmholtz determinant of a lossy material, the following holds

$$
\lim _{w \rightarrow \infty} \frac{D\left(\boldsymbol{s}_{a}+w \boldsymbol{s}_{b}\right)}{w^{4}} \neq 0 .
$$

Proof: It is easily checked that

$$
\lim _{w \rightarrow \infty} \frac{D\left(\boldsymbol{s}_{a}+w \boldsymbol{s}_{b}\right)}{w^{4}}=\varepsilon_{b b} \mu_{b b}-\zeta_{b b} \xi_{b b},
$$

with

$$
\chi_{b b}=s_{b} \cdot \overline{\bar{\chi}} \cdot s_{b}^{T}, \forall \chi \in\{\varepsilon, \mu, \zeta, \xi\} .
$$

Now define a two dimensional space of vectors $V$ of the form

$$
\boldsymbol{V}=\left[\begin{array}{cc}
\boldsymbol{s}_{b}^{T} & \mathbf{0} \\
\mathbf{0} & \boldsymbol{s}_{b}^{T}
\end{array}\right] \cdot\left[\begin{array}{l}
a \\
b
\end{array}\right]
$$

where $a$ and $b$ are complex scalars and the matrix has size $6 \times 2$. Applying the lossyness criterium (17) to these 'special' vectors yields

$$
\Im\left\{\left[\begin{array}{ll}
a^{H} & b^{H}
\end{array}\right] \cdot\left[\begin{array}{cc}
\varepsilon_{b b} & \xi_{b b} \\
\zeta_{b b} & \mu_{b b}
\end{array}\right] \cdot\left[\begin{array}{l}
a \\
b
\end{array}\right]\right\}<0, \forall\left[\begin{array}{l}
a \\
b
\end{array}\right] \in \mathbb{C}^{2} \backslash\{\mathbf{0}\} .
$$

This statement implies that the matrix in the middle of (33) is not singular, which in turns means that its determinant $\varepsilon_{b b} \mu_{b b}-$ $\zeta_{b b} \xi_{b b}$ is nonzero, concluding the proof.

The limit in (29) is essentially the coefficient of $\lambda^{4}$ in the polynomial root finding problem (28). Therefore, the fact that Theorem 3.3 shows that this coefficient is nonzero implies 
that there exist exactly four roots, i.e. solutions to (28). This result is quite remarkable because it establishes a connection between two concepts that are at first sight unrelated (lossyness and the guaranteed existence of four solutions).

The result from Theorem 3.3 can be refined further:

Theorem 3.4: Let $\boldsymbol{s}_{a}$ and $\boldsymbol{s}_{b}$ be $\in \mathbb{R}^{3}$, and $D(\boldsymbol{s})$ be the Helmholtz determinant of a lossy material. Then of the four solutions $\lambda_{n}, n \in[1,4]$ of equation (28), two have a strictly positive imaginary part, and two have a strictly negative imaginary part.

Proof: It is well-known that the $p$ roots of a polynomial of degree $p$ are continuous functions of the polynomial's $p+1$ coefficients [14], [15], except when the coefficient of the $p$ th degree term is zero. In our case, the polynomial under scrutiny is the left hand side of equation (28), is of degree four and, inside the set of lossy materials, has a nonzero fourth degree coefficient. Also, the polynomial coefficients are themselves polynomials in the elements of the material matrix $M$ and the vectors $\boldsymbol{s}_{a}$ and $\boldsymbol{s}_{b}$. Therefore, crucially, the solutions $\lambda_{n}$ of equation (28) are continuous functions of $\mathrm{M}, \boldsymbol{s}_{a}$ and $\boldsymbol{s}_{b}$, as long as the material remains lossy. By examining the following simple lossy material

$$
\mathrm{M}_{s}=\left[\begin{array}{cc}
-j \mathbb{1} & 0 \\
0 & -j \mathbb{1}
\end{array}\right]
$$

with $\boldsymbol{s}_{a}=\mathbf{0}$ and $\boldsymbol{s}_{b}=[0,0,1]$, it is easily shown that

$$
\lambda_{1}=j, \lambda_{2}=j, \lambda_{3}=-j, \lambda_{4}=-j .
$$

Clearly, there are two roots with positive imaginary part, and two roots with negative imaginary part. Now, if $\mathrm{M}, \boldsymbol{s}_{a}$ and $s_{b}$ are continuously varied towards other values, whilst remaining lossy, the number of roots with a positive imaginary part must remain constant. Indeed, if the positive imaginary part would somehow become negative, it should have passed through zero because of the continuity of the roots. However, such a transit through zero is forbidden since that would violate Theorem (3.2). Summarizing, we have that roots conserve the sign of their imaginary part under continuous variation of the elements of $\mathrm{M}, \boldsymbol{s}_{a}$ and $\boldsymbol{s}_{b}$, as long as the material remains lossy. Finally, it remains to be shown that any lossy material can be reached, starting from $\mathrm{M}_{s}$, by means of a continuous path that lies entirely inside the set of lossy materials. Because of the convexity of the set of lossy materials (Theorem 3.1), it is clear that a simple straight path between the two points satisfies all these requirements.

The theorems proved in this Section will provide a firm theoretical footing for the rest of this paper. In particular, they preclude many pathological cases in the numerical computation of the BSGF expansion coefficients. Because of this, the resulting computation algorithm will be very robust.

\section{COMPUTATION OF THE CHEB YSheV EXPANSION COEFFICIENTS}

For reasons explained in the introduction, the BSGF will be expanded into Chebyshev polynomials

$$
G(\boldsymbol{r})=\sum_{p_{x}, p_{y}, p_{z}=0}^{\infty} C_{\boldsymbol{p}} T_{p_{x}}\left(\frac{x-c_{x}}{a_{x}}\right) T_{p_{y}}\left(\frac{y-c_{y}}{a_{y}}\right) T_{p_{z}}\left(\frac{z-c_{z}}{a_{z}}\right),
$$

for all $\boldsymbol{r}$ in the validity range $\square_{c, a}$. The range $\square_{c, a}$ is a cuboidal region with center $c$ and sides $2 a_{x}, 2 a_{y}$ and $2 a_{z}$ along the three coordinate axes. Integration over this range will henceforth be denoted by

$$
\int_{\square_{\boldsymbol{c}, \boldsymbol{a}}} f(\boldsymbol{r}) \mathrm{d} \boldsymbol{r}=\int_{c_{x}-a_{x}}^{c_{x}+a_{x}} \int_{c_{y}-a_{y}}^{c_{y}+a_{y}} \int_{c_{z}-a_{z}}^{c_{z}+a_{z}} f(\boldsymbol{r}) \mathrm{d} \boldsymbol{r} .
$$

The computation of the Chebyshev expansion coefficients $C_{p}$ is done by projecting the BSGF onto the Chebyshev polynomials. This leads to the following expression for the Chebyshev expansion coefficients:

$$
C_{\boldsymbol{p}}=\frac{\alpha_{p_{x}} \alpha_{p_{y}} \alpha_{p_{z}}}{\pi^{3} a_{x} a_{y} a_{z}} \int_{\square_{c, a}} G(\boldsymbol{r}) H(\boldsymbol{p}, \boldsymbol{a}, \boldsymbol{r}-\boldsymbol{c}) \mathrm{d} \boldsymbol{r},
$$

with $\alpha_{p}=2-\delta_{p, 0}$ and

$$
H(\boldsymbol{p}, \boldsymbol{a}, \boldsymbol{r})=\frac{T_{p_{x}\left(\frac{x}{a_{x}}\right)}}{\sqrt{1-\left(\frac{x}{a_{x}}\right)^{2}}} \frac{T_{p_{y}}\left(\frac{y}{a_{y}}\right)}{\sqrt{1-\left(\frac{y}{a_{y}}\right)^{2}}} \frac{T_{p_{z}}\left(\frac{z}{a_{z}}\right)}{\sqrt{1-\left(\frac{z}{a_{z}}\right)^{2}}} .
$$

Using the definition of the BSGF (16), the expansion coefficients become

$$
C_{\boldsymbol{p}}=\frac{\alpha_{p_{x}} \alpha_{p_{y}} \alpha_{p_{z}}}{8 \pi^{6} a_{x} a_{y} a_{z}} \int_{\square_{c, a}} \int_{\mathbb{R}^{3}} \frac{e^{j \boldsymbol{s} \cdot \boldsymbol{r}}}{D(\boldsymbol{s})} H(\boldsymbol{p}, \boldsymbol{a}, \boldsymbol{r}-\boldsymbol{c}) \mathrm{d} \boldsymbol{s} \mathrm{d} \boldsymbol{r} .
$$

Fubini's theorem states that the integrations over $r$ and $s$ can be interchanged if

$$
\int_{\square_{c, a}} \int_{\mathbb{R}^{3}}\left|\frac{e^{j \boldsymbol{s} \cdot \boldsymbol{r}}}{D(\boldsymbol{s})} H(\boldsymbol{p}, \boldsymbol{c}, \boldsymbol{a}, \boldsymbol{r}-\boldsymbol{c})\right| \mathrm{d} \boldsymbol{s} \mathrm{d} \boldsymbol{r}<\infty .
$$

Since the absolute value of the exponential is 1 , and the absolute value of the Chebyshev polynomials is always less than or equal to 1 , this criterium simplifies to

$$
\int_{\square_{\boldsymbol{c}, \boldsymbol{a}}}|H([0,0,0], \boldsymbol{a}, \boldsymbol{r}-\boldsymbol{c})| \mathrm{d} \boldsymbol{r} \int_{\mathbb{R}^{3}}\left|\frac{1}{D(\boldsymbol{s})}\right| \mathrm{d} \boldsymbol{s}<\infty,
$$

which is true if the Helmholtz determinant never becomes zero for real $s$. By virtue of Theorem 3.2, this requirement is always satisfied if the material is lossy. An intuitive explanation is found by looking at the fact that the BSGF is exponentially decaying in all directions in a lossy material. Therefore, it can be concluded that its Fourier transform, i.e. $\frac{1}{D(s)}$, must be a smooth function. The Helmholtz determinant can of course become zero for lossless materials (most notably an isotropic lossless material), or materials that are lossy only for certain propagation directions and polarizations. In such a case, proving that the integrations can be interchanged is more difficult, if not impossible in general. However, since it was assumed in the introduction that the materials under consideration are lossy, this case will not be considered further. 
Summarizing, the two integrations in (40) can be interchanged because the material is lossy. Hence, the Chebyshev expansion coefficients can be written as

$$
C_{\boldsymbol{p}}=\frac{\alpha_{p_{x}} \alpha_{p_{y}} \alpha_{p_{z}}}{8 \pi^{6} a_{x} a_{y} a_{z}} \int_{\mathbb{R}^{3}} \int_{\square_{\boldsymbol{c}, \boldsymbol{a}}} H(\boldsymbol{p}, \boldsymbol{a}, \boldsymbol{r}-\boldsymbol{c}) e^{j \boldsymbol{s} \cdot \boldsymbol{r}} \mathrm{d} \boldsymbol{r} \frac{1}{D(\boldsymbol{s})} \mathrm{d} \boldsymbol{s} .
$$

The inner integral can now be evaluated analytically by means of

$$
\int_{-1}^{1} \frac{T_{n}(x)}{\sqrt{1-x^{2}}} e^{j x v} \mathrm{~d} x=j^{n} \pi J_{n}(v)
$$

which yields

$$
C_{\boldsymbol{p}}=j^{p_{x}+p_{y}+p_{z}} \frac{\alpha_{p_{x}} \alpha_{p_{y}} \alpha_{p_{z}}}{8 \pi^{3}} \int_{\mathbb{R}^{3}} \frac{F(\boldsymbol{p}, \boldsymbol{c}, \boldsymbol{a}, \boldsymbol{s})}{D(\boldsymbol{s})} \mathrm{d} \boldsymbol{s},
$$

with

$$
F(\boldsymbol{p}, \boldsymbol{c}, \boldsymbol{a}, \boldsymbol{s})=e^{j \boldsymbol{c} \cdot \boldsymbol{s}} J_{p_{x}}\left(s_{x} a_{x}\right) J_{p_{y}}\left(s_{y} a_{y}\right) J_{p_{z}}\left(s_{z} a_{z}\right) .
$$

Leveraging the fact that the Helmholtz determinant is a polynomial of degree four, the three dimensional integration in (45) can be reduced to a two dimensional integration by means of complex contour integration. To this end, let us fix the value of $s_{x}$ and $s_{y}$ and find the roots of the Helmholtz determinant in the $s_{z}$ direction. This boils down to solving equation (28) with $s_{a}=\left[s_{x}, s_{y}, 0\right]$ and $s_{b}=[0,0,1]$. Theorem 3.3 guarantees the existence of exactly four roots, which are denoted by $\lambda_{n}\left(s_{x}, s_{y}\right), \forall n \in[1,4]$, i.e.

$$
D\left(\left[s_{x}, s_{y}, \lambda_{n}\left(s_{x}, s_{y}\right)\right]\right)=0, \forall s_{x}, s_{y} \in \mathbb{R}, \forall n \in[1,4],
$$

Now, using these four roots, one over the Helmholtz determinant can be written as a partial fraction expansion

$$
\frac{1}{D(s)}=\frac{1}{D_{z}} \prod_{n=1}^{4} \frac{1}{s_{z}-\lambda_{n}\left(s_{x}, s_{y}\right)}=\frac{1}{D_{z}} \sum_{n=1}^{4} \frac{a_{n}\left(s_{x}, s_{y}\right)}{s_{z}-\lambda_{n}\left(s_{x}, s_{y}\right)},
$$

with the highest degree coefficient

$$
D_{z}=\varepsilon_{z z} \mu_{z z}-\zeta_{z z} \xi_{z z},
$$

and the partial fraction coefficients

$$
a_{n}\left(s_{x}, s_{y}\right)=\prod_{\substack{m=1 \\ m \neq n}}^{4} \frac{1}{\lambda_{m}\left(s_{x}, s_{y}\right)-\lambda_{n}\left(s_{x}, s_{y}\right)} .
$$

According to Theorem 3.3, $D_{z}$ is guaranteed to be nonzero for lossy materials. This is necessary for (48) to be valid. However, it is not enough, since nothing prevents the multiplicity of these roots to be greater than one (this is for example the case in an isotropic material). In a case where the multiplicity of the roots is greater than one, the partial fraction coefficients (50) become infinite, which is to be avoided in numerical computations. Therefore, some special techniques are required to handle this. These will be detailed in the next Section.

Finally, the contour integration can be performed. To do this, assume that $0<a_{z}<c_{z}$, i.e. the entire expansion range $\square_{c, \boldsymbol{a}}$ is located above the $x y$-plane. Under this assumption, it can be shown that $e^{j c_{z} \cdot s_{z}} J_{p_{z}}\left(s_{z} a_{z}\right)$ converges to zero in an exponential fashion when $s_{z}$ is moved towards $j \infty$. Hence, leveraging the fact that $F(\boldsymbol{p}, \boldsymbol{c}, \boldsymbol{a}, \boldsymbol{s})$ is a holomorphic function of $s$, the residue theorem [16] can be applied to compute the integral with respect to $s_{z}$ in (45). This computation yields

$$
\begin{aligned}
& \int_{\mathbb{R}^{3}} \frac{F(\boldsymbol{p}, \boldsymbol{c}, \boldsymbol{a}, \boldsymbol{s})}{D(\boldsymbol{s})} \mathrm{d} \boldsymbol{s} \\
& \quad=\frac{2 \pi j}{D_{z}} \int_{\mathbb{R}^{2}} \sum_{\substack{n=1 \\
\Im \lambda_{n}>0}}^{4} a_{n} F\left(\boldsymbol{p}, \boldsymbol{c}, \boldsymbol{a},\left[s_{x}, s_{y}, \lambda_{n}\right]\right) \mathrm{d} s_{x} \mathrm{~d} s_{y},
\end{aligned}
$$

where the dependence on $s_{x}$ and $s_{y}$ was removed for brevity and clarity. Due to the use of the residue theorem, the sum in equation (51) runs only over those roots that have a positive imaginary part. Theorem (3.4) states that exactly two of the four roots have a strictly positive imaginary part, while the other two have a strictly negative imaginary part. Therefore, the summation actually only runs over two roots.

Equation (51) can now be used to compute the Chebyshev expansion coefficients $C_{\boldsymbol{p}}$ up to arbitrary expansion order $P$ using only a two dimensional numerical integration. In addition, the integrand is converging to zero exponentially fast, when $s_{x}$ and $s_{y}$ are moved away from the origin. It should also be pointed out that this reduction of a three dimensional integration to a two dimensional integration is not limited to expansion ranges $\square_{c, a}$ located entirely above the $x y$-plane. In fact, an analytical contour integration (possibly over other coordinates than $s_{z}$ ) can be performed for any rectangular expansion range that does not include or touch the origin. The data structure that patches together all the expansion ranges into a coverage of $\mathbb{R}^{3}$ therefore needs to make sure that the requirement of not touching the origin is always met.

\section{Handling a Root with Multiplicity Two}

In the previous Section, the Chebyshev expansion coefficients were expressed as a two dimensional integral, of which the integrand is a sum over two roots of the Helmholtz determinant. It was noted that the partial fraction expansion of one over the Helmholtz determinant exhibits problematic numerical behavior if two of its roots are identical. It is worthwhile to point out that this problem can occur for even the simplest material (i.e. an isotropic material). Even if two roots are merely close together, a numerical cancelation due to rounding error degrades the result.

To solve this issue, one needs to realize that the summation over the roots in (51) has a removable singularity. Once this singularity has been removed, it becomes an entire function in the roots $\lambda_{n}\left(s_{x}, s_{y}\right)$. In this Section, the removable singularity will be isolated in three auxiliary functions, for which it is easier to remove the singularity. Hence, the final expression can be evaluated in a numerically stable way.

Starting from the summation in (51), and moving everything that does not depend on $n$ outside of the summation sign, the following quantity needs to be computed

$$
\sum_{\substack{n=1 \\ \Im \lambda_{n}>0}}^{4} a_{n} e^{j c_{z} \lambda_{n}} J_{p_{z}}\left(a_{z} \lambda_{n}\right)
$$


Suppose that $\lambda_{1}$ and $\lambda_{2}$ are the only two roots that have a positive imaginary part. Then the following partial fraction expansion can be made

$$
\begin{aligned}
\prod_{n=1}^{4} \frac{1}{s_{z}-\lambda_{n}} & =\frac{d_{1} s_{z}+b 1}{\left(s_{z}-\lambda_{1}\right)\left(s_{z}-\lambda_{2}\right)}+\frac{d_{2} s_{z}+b 2}{\left(s_{z}-\lambda_{3}\right)\left(s_{z}-\lambda_{4}\right)}, \\
d_{1} & =-\frac{1}{Q}\left(\lambda_{1}-\lambda_{4}-\lambda_{3}+\lambda_{2}\right) \\
b_{1} & =\frac{1}{Q}\left(\lambda_{1}^{2}+\lambda_{1} \lambda_{2}+\lambda_{2}^{2}-\lambda_{1} \lambda_{4}\right. \\
\left.\quad-\lambda_{3} \lambda_{1}-\lambda_{2} \lambda_{4}-\lambda_{3} \lambda_{2}+\lambda_{3} \lambda_{4}\right) & \\
Q & =\left(\lambda_{2}-\lambda_{4}\right)\left(\lambda_{2}-\lambda_{3}\right)\left(\lambda_{1}-\lambda_{3}\right)\left(\lambda_{1}-\lambda_{4}\right)
\end{aligned}
$$

A crucial point is that $Q^{-1}$ (and therefore also $d_{1}$ and $b_{1}$ ) can be computed without numerical problems because only differences appear between roots that have an imaginary part of different sign. Also, the expressions for $d_{2}$ and $b_{2}$ are not given since they do not contribute to the contour integral (51). Using (53), the summation (52) can be written as

$$
\begin{aligned}
(52)= & \frac{d_{1}}{\lambda_{1}-\lambda_{2}}\left[\lambda_{1} e^{j c_{z} \lambda_{1}} J_{p_{z}}\left(a_{z} \lambda_{1}\right)-\lambda_{2} e^{j c_{z} \lambda_{2}} J_{p_{z}}\left(a_{z} \lambda_{2}\right)\right] \\
& +\frac{b_{1}}{\lambda_{1}-\lambda_{2}}\left[e^{j c_{z} \lambda_{1}} J_{p_{z}}\left(a_{z} \lambda_{1}\right)-e^{j c_{z} \lambda_{2}} J_{p_{z}}\left(a_{z} \lambda_{2}\right)\right] .
\end{aligned}
$$

Clearly, the singularity for $\lambda_{1} \rightarrow \lambda_{2}$ is removable. Despite this, large rounding errors are introduced when evaluating this expression numerically if the two roots are close to each other. To remove this problem, we propose to isolate the removable singularity in three functions

$$
\begin{aligned}
E(x, y) & =\frac{e^{j x}-e^{j y}}{x-y}, \\
K(x, y) & =\frac{x e^{j x}-y e^{j y}}{x-y}, \\
S_{p}(x, y) & =\frac{J_{p}(x)-J_{p}(y)}{x-y},
\end{aligned}
$$

which all have removable singularities. In Appendix A, algorithms are proposed to compute these functions in a numerically robust way. Using these functions, it is possible to rewrite (52) in a numerically satisfactory form:

$$
\begin{aligned}
& \frac{d_{1}}{2}\left[J_{p_{z}}\left(a_{z} \lambda_{1}\right)+J_{p_{z}}\left(a_{z} \lambda_{2}\right)\right] K\left(c_{z} \lambda_{1}, c_{z} \lambda_{2}\right) \\
& +\frac{d_{1} a_{z}}{2}\left[\lambda_{1} e^{j c_{z} \lambda_{1}}+\lambda_{2} e^{j c_{z} \lambda_{2}}\right] S_{p_{z}}\left(a_{z} \lambda_{1}, a_{z} \lambda_{2}\right) \\
& +\frac{b_{1} c_{z}}{2}\left[J_{p_{z}}\left(a_{z} \lambda_{1}\right)+J_{p_{z}}\left(a_{z} \lambda_{2}\right)\right] E\left(c_{z} \lambda_{1}, c_{z} \lambda_{2}\right) \\
& +\frac{b_{1} a_{z}}{2}\left[e^{j c_{z} \lambda_{1}}+e^{j c_{z} \lambda_{2}}\right] S_{p_{z}}\left(a_{z} \lambda_{1}, a_{z} \lambda_{2}\right) .
\end{aligned}
$$

Using the versions of $E, K$ and $S_{p}$ defined in Appendix A, this alternative form of (52) is free of removable singularities. Hence it does not suffer from catastrophic loss of precision. Expression (61) therefore removes the main class of numerical problems in the computation of the Chebyshev expansion coefficients. In fact, we have been unable to identify any further pathways to divisions by zero or the generation of NaNs. Of course, for certain pathological materials, the computation could become very slow or unexpected numerical cancelations may occur for certain field components. However, no 'hard' breakdowns will occur, which makes the code very robust.

\section{Computation of the BSGF Derivatives}

One of the main advantages of the Chebyshev expansion for storing the BSGF is that it also allows the derivatives to be computed with great ease and precision. Indeed, if a derivative with respect to for example $x$ of (36) is needed, it suffices to just evaluate the derivative of the first Chebyshev polynomial with respect to $x$. The $n$th derivative of a Chebyshev polynomial is easily computed recursively using

$2 \frac{\mathrm{d}^{n}}{\mathrm{~d} u^{n}} T_{p}(u)=\frac{1}{p+1} \frac{\mathrm{d}^{n+1}}{\mathrm{~d} u^{n+1}} T_{p+1}(u)-\frac{1}{p-1} \frac{\mathrm{d}^{n+1}}{\mathrm{~d} u^{n+1}} T_{p-1}(u)$.

As starting value for this recurrence in the derivative degree $n$, the Chebyshev polynomials themselves are required. These can be computed using either the recurrence

$$
T_{p+1}(u)=2 u T_{p}(u)-T_{p-1}(u),
$$

or the explicit formula

$$
T_{p}(u)=\cos (p \arccos u) .
$$

Clearly, taking derivatives of a Chebyshev series is easily implemented. However, one should be aware of the fact that the Chebyshev polynomials oscillate more quickly at the edges of the range $[-1,1]$. Therefore, their derivatives have a larger magnitude at those edges. In fact, the derivatives of the Chebyshev polynomials reach their maximum magnitude at the points 1 and -1 , with extremal value $\kappa_{p, n}$ given by

$$
\kappa_{p, n}=\left.\frac{\mathrm{d}^{n}}{\mathrm{~d} u^{n}} T_{p}(u)\right|_{u=1}=\prod_{l=0}^{n-1} \frac{p^{2}-l^{2}}{2 l+1} .
$$

Because these values increase quickly as a function of $p$, the Chebyshev series (36) converges more slowly if more and more derivatives are taken. Because of this slower convergence, Chebyshev expansion coefficients that hardly play a role in the evaluation of the BSGF can become very important for the accuracy of the derivatives of the BSGF. This is one of the reasons why the expansion in Chebyshev polynomials is so advantageous: using existing software for the accurate evaluation of the Bessel functions, it is possible to accurately compute even extremely small Chebyshev expansion coefficients by means of (51). These small coefficients do not contribute much to the BSGF itself, but can be required for the accurate evaluation of its derivatives.

\section{STORAGE OF THE BSGF}

\section{A. Storage Data Structure}

In the previous Section, the procedure for computing the Chebyshev expansion coefficients was explained. However, it 
was also found that the complex contour integration can only be done if the rectangular expansion range does not touch the origin. The data structure used to store all the Chebyshev expansion coefficients should accommodate this requirement, which means that the collection of expansion ranges will cover at most $\mathbb{R}^{3} \backslash\{\mathbf{0}\}$. Generally, this is not a problem because the Green's dyadics will never be evaluated in the singularity anyway.

The storage data structure proposed in this paper consists of a multilevel tree of cubic boxes, augmented with a given Chebyshev expansion order $P$ (which is infinite in equation (36), but finite in practice). An example tree is depicted in Figure 2. Each box has a level $l \in \mathbb{Z}$ and an index vector $n \in$ $\mathbb{Z}^{3}$. By definition the location of the center of a box is given by $3^{l} \boldsymbol{n}$, and its side is $3^{l}$. Clearly, on each level, the box with $\boldsymbol{n}=\mathbf{0}$ contains the origin, whereas the other boxes do not even touch the origin. The expansion range associated with each box is simply the entire box, i.e. $\boldsymbol{c}=3^{l} \boldsymbol{n}$ and $\boldsymbol{a}=\frac{1}{2} 3^{l}[1,1,1]$. The Chebyshev expansion associated with a box is the set of Chebyshev coefficients $C_{\boldsymbol{p}}, \forall p_{x}, p_{y}, p_{z} \leq P$, as computed for the box's expansion range. A box is called a valid expansion box if its Chebyshev expansion attains the desired accuracy $\epsilon$ over the whole expansion range. To determine whether the desired accuracy has been achieved, a criterium will be derived at the end of this Section.

When the evaluation of the BSGF in a point $r$ is requested for the first time, the data structure is still empty. It then starts by constructing the smallest $\boldsymbol{n}=\mathbf{0}$ box that contains $\boldsymbol{r}$. Let this box be called the 'root' box and let $L$ be its level. Since no Chebyshev expansion can be computed for this box, the data structure will also construct the level $L-1$ level box that contains $r$ and compute the Chebyshev expansion. This level $L-1$ box will be called a 'child' of the level $L$ box. Since the expansion order has been fixed a priori at $P$, one cannot be certain that the Chebyshev expansion will be accurate enough. Therefore, the error of the Chebyshev expansion is computed. If the error is small enough, the process stops there. If the error is too large, a level $L-2$ box is constructed that is the child of the level $L-1$ box and also contains $\boldsymbol{r}$, and so on. Eventually, the box for which the Chebyshev expansion is computed, becomes so small, and the convergence of the Chebyshev expansion so rapid, that the error becomes small enough and the process halts in a valid expansion box. Then, the Chebyshev expansion is simply evaluated in $r$ and the value returned.

If, after this initial evaluation, an evaluation in another point $\boldsymbol{r}^{\prime}$ is requested, the data structure first looks up the smallest box in which $\boldsymbol{r}^{\prime}$ is contained. If no box contains $\boldsymbol{r}^{\prime}$, this means that the root box needs to be moved to a higher level than $L$. If it is contained in a certain box, then this can either be a valid expansion box or not. If it is a valid expansion box, the expansion is again evaluated and the value returned. If it is not a valid expansion box, a string of child boxes is again constructed until a valid expansion box is found. In this way, valid expansion boxes never have child boxes, which makes sure that unneeded refinement of the boxes is prevented. Also, any evaluation point other than $\mathbf{0}$ can be handled, and the error is controllable.

\section{B. Accuracy Criterium}

To complete the adaptive scheme described in the above, a criterium must be constructed to determine whether a given Chebyshev expansion is accurate. One of the most natural ways to construct such a criterium is to use the differential equation of the BSGF:

$$
D(-j \boldsymbol{\nabla}) G(\boldsymbol{r})=0, \forall \boldsymbol{r} \in \mathbb{R}^{3} \backslash\{\mathbf{0}\},
$$

which is easily derived from the integral representation (16). Because the Chebyshev expansion (36) has been truncated to order $P$, the BSGF after truncation $G_{P}(\boldsymbol{r})$ will not be an exact solution of differential equation (66). Instead, it leads to a function

$$
\Delta(\boldsymbol{r})=D(-j \nabla) G_{P}(\boldsymbol{r}),
$$

that can be used to estimate the error introduced by truncating the series. To do this, note that the right hand side of (67) is composed of 35 terms, each corresponding to a term in the fourth-degree polynomial that is the Helmholtz determinant. When computing their sum, a lot of cancelation takes place. In fact, the more cancelation, the better the accuracy of $G_{P}(\boldsymbol{r})$, since it better satisfies the differential equation (66). In numerical analysis, the relevant quantity for measuring the amount of cancelation in a real summation is the condition number [17]:

$$
\mathrm{CN}\left[\sum_{i} x_{i}\right]=\frac{\sum_{i}\left|x_{i}\right|}{\left|\sum_{i} x_{i}\right|}, x_{i} \in \mathbb{R} .
$$

This condition number expresses how much the magnitude of the sum differs from the magnitude it could have had if all the signs would have been the same. For such a real summation, one is therefore naturally led to a convergence criterium of the form

$$
\mathrm{CN}\left[\sum_{i} x_{i}\right]>\frac{1}{\epsilon}
$$

For a complex summation, this criterium should be applied to both the real and imaginary part of the sum, since they are summed independently. In our case, however, the real part of $\Delta(\boldsymbol{r})$ can sometimes be much smaller than the imaginary part or vice versa. In such a case, a high relative error on the small part is perfectly acceptable, but it will not pass criterium (69). A heuristic solution to this problem is to judge the real and imaginary part together by means of

$$
\mathrm{CN}^{\prime}\left[\sum_{i} z_{i}\right]=\frac{\sqrt{\left[\sum_{i}\left|\Re z_{i}\right|\right]^{2}+\left[\sum_{i}\left|\Im z_{i}\right|\right]^{2}}}{\left|\sum_{i} z_{i}\right|}, z_{i} \in \mathbb{C} .
$$

This modified condition number again expresses how much the magnitude of the sum differs from the magnitude it could have had if all the signs would have been the same. It therefore naturally leads to the error criterium that is used in our implementation:

$$
\mathrm{CN}^{\prime}[\Delta(\boldsymbol{r})]>\frac{1}{\epsilon}, \forall \boldsymbol{r} \in \square_{\boldsymbol{c}, \boldsymbol{a}}
$$

Here, the condition number is understood to be computed with respect to the summation over the 35 terms of the Helmholtz 
determinant. An expansion is considered sufficiently accurate if this inequality is satisfied.

Criterium (71) has the advantage that both the lowest-order derivatives and the highest-order derivatives are taken into account. This is necessary, because the lowest-order derivatives are not sensitive to errors in the high-order Chebyshev coefficients. In addition, it is free of absolute quantities, i.e. the multiplication of the BSGF with a real constant does not influence the criterium. A drawback is the lack of relative error guarantees on each of the 35 terms of the Helmholtz determinant separately. However, such guarantees would be hard to achieve because these terms can become exactly zero. In addition, criterium (71) gives good results in practice, as will be shown in the numerical results Section VIII.

Concerning the choice of test points $r$ in (71), numerical tests have shown that the error of the Chebyshev expansion is usually highest in the eight vertices of the expansion range, due to the presence of the derivatives and the fact that the derived Chebyshev polynomials reach their maximum magnitude there. Hence, for the next Section, the set of points to test (71) in was chosen to be the set of these eight vertices.

\section{NUMERICAL RESULTS}

To test the proposed computation and storage algorithms, a number of numerical tests will be performed. First, the BSGF will be compared with its analytically known form for biisotropic materials. Next, a similar test will be performed, but for a uniaxial material. Finally, a test will be done with a completely general lossy bianisotropic material.

For all these tests, the relative material parameters

$$
\overline{\bar{\varepsilon}}_{r}=\frac{\overline{\bar{\varepsilon}}}{\varepsilon_{0}}, \overline{\bar{\mu}}_{r}=\frac{\overline{\bar{\mu}}}{\mu_{0}}, \overline{\bar{\zeta}}_{r}=\frac{\overline{\bar{\zeta}}}{\sqrt{\varepsilon_{0} \mu_{0}}}, \overline{\bar{\xi}}_{r}=\frac{\overline{\bar{\xi}}}{\sqrt{\varepsilon_{0} \mu_{0}}},
$$

will be used instead of the actual values. This does not reduce the generality of the results because the following connection can be established:

$$
G(\boldsymbol{r})=\frac{G_{\mathrm{rel}}\left(\boldsymbol{r} \sqrt{\varepsilon_{0} \mu_{0}}\right)}{\sqrt{\varepsilon_{0}^{3} \mu_{0}^{3}}},
$$

where $G_{\text {rel }}(\boldsymbol{r})$ is the BSGF for a material with material matrix

$$
\mathrm{M}_{r}=\left[\begin{array}{ll}
\overline{\bar{\varepsilon}}_{r} & \overline{\bar{\xi}}_{r} \\
\overline{\bar{\zeta}}_{r} & \overline{\bar{\mu}}_{r}
\end{array}\right] \text {. }
$$

Clearly, if $G_{\text {rel }}(\boldsymbol{r})$ and its derivatives can be accurately computed, so can the BSGF with the actual material parameters. Therefore, we can limit ourselves to the testing of $G_{\text {rel }}(\boldsymbol{r})$. For the tests below, the accuracy of the adaptive integration routines and the storage tree subdivision strategy were both set to $10^{-10}$ and the truncation order $P$ was set to 20. The coordinate range in which the tests are done is $\boldsymbol{r}=[x, 0,1], \forall x \in[-10,10]$.

\section{A. Bi-isotropic Material}

For a bi-isotropic material, the material dyadics are given by

$$
\overline{\bar{\varepsilon}}_{r}=\varepsilon_{r} \mathbb{1}, \overline{\bar{\mu}}_{r}=\mu_{r} \mathbb{1}, \overline{\bar{\zeta}}_{r}=\zeta_{r} \mathbb{1}, \overline{\bar{\xi}}_{r}=\xi_{r} \mathbb{1} .
$$

For such a material, the Helmholtz determinant can be simplified considerably and factorizes into

$$
D(s)=\left(\mu_{r} \varepsilon_{r}-\xi_{r} \zeta_{r}\right)\left(s^{2}-s_{+}^{2}\right)\left(s^{2}-s_{-}^{2}\right) .
$$

Here, $s$ denotes the norm of the slowness vector $\|s\|$. Furthermore, the constants $s_{+}^{2}$ and $s_{-}^{2}$ are given by

$s_{ \pm}^{2}=\frac{1}{2}\left[2 \mu_{r} \varepsilon_{r}-\xi_{r}^{2}-\zeta_{r}^{2} \pm \sqrt{\left(\xi_{r}^{2}-\zeta_{r}^{2}\right)^{2}-4 \mu_{r} \varepsilon_{r}\left(\zeta_{r}-\xi_{r}\right)^{2}}\right]$.

For this material, the BSGF can be analytically derived as

$$
G_{\mathrm{rel}}(\boldsymbol{r})=\frac{1}{\mu_{r} \varepsilon_{r}-\xi_{r} \zeta_{r}} \frac{e^{-i s_{+} r}-e^{-i s_{-} r}}{4 \pi r\left(s_{+}^{2}-s_{-}^{2}\right)},
$$

where $r=\|\boldsymbol{r}\|$. For the computation of $s_{ \pm}$from $s_{ \pm}^{2}$, the root that yields an exponentially decreasing BSGF for increasing $r$ should be chosen. This is required to get results consistent with the BSGF defined by means of the inverse Fourier transform (16), which always yields an exponentially decreasing BSGF for lossy materials.

For the comparison between (78) and its numerically computed counterpart, we chose the material parameters somewhat arbitrarily as

$$
\begin{gathered}
\varepsilon_{r}=1.0-0.1 j, \\
\zeta_{r}=0.185+0.055 j, \\
\xi_{r}=0.275-0.085 j, \\
\mu_{r}=2.0-0.05 j .
\end{gathered}
$$

This material is lossy and nonreciprocal. Figure 1 shows a comparison between the numerically computed BSGF and its analytical form. Figure 2 shows the boxes that were selected by the storage tree. To show the accuracy of the derivatives, the fourth derivatives with respect to the $x, y$ and $z$-coordinate were plotted. It is clear that, if the fourth derivative is accurately computed, the lower-order derivatives will also be accurate. Excellent agreement is observed, as evidenced by the very low relative error between the numerical and analytical result.

Some gradual rises and sharp drops in the relative error can be observed. This is due to the fact that, if two boxes have the same size, the error is usually higher in the box nearer to the origin than in the one farther away. If a box with a certain size is too close, the adaptive subdivision mechanism of the storage tree decides that it cannot guarantee the target accuracy and it decreases the box size (i.e. its level). For the bi-isotropic medium (79), this reduction in box size happens at positions $x= \pm 4.5$ and $x= \pm 1.5$, which can also be seen in Figure 2.

\section{B. Uniaxial Material}

In this second test, the following uniaxial material is considered:

$$
\begin{gathered}
\overline{\bar{\varepsilon}}_{r}=\left[\begin{array}{ccc}
\varepsilon_{t} & 0 & 0 \\
0 & \varepsilon_{t} & 0 \\
0 & 0 & \varepsilon_{z}
\end{array}\right], \quad \overline{\bar{\mu}}_{r}=\left[\begin{array}{ccc}
\mu_{t} & 0 & 0 \\
0 & \mu_{t} & 0 \\
0 & 0 & \mu_{z}
\end{array}\right], \\
\overline{\bar{\zeta}}_{r}=0, \quad \overline{\bar{\xi}}_{r}=0 .
\end{gathered}
$$




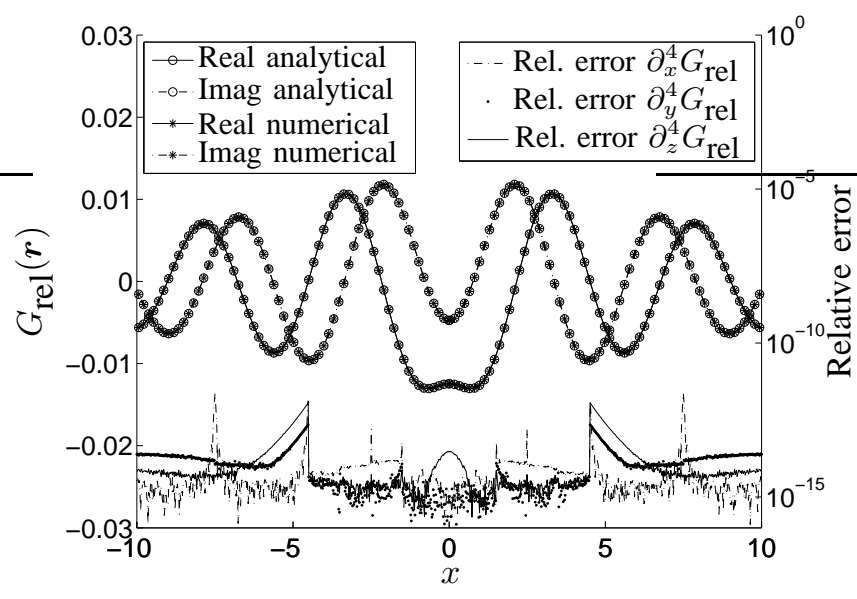

Fig. 1. A comparison, for the bi-isotropic medium (79), between the numerically computed BSGF and its analytical counterpart (78). The right axis pertains to the relative error between the fourth derivatives of the numerical and analytical BSGF.

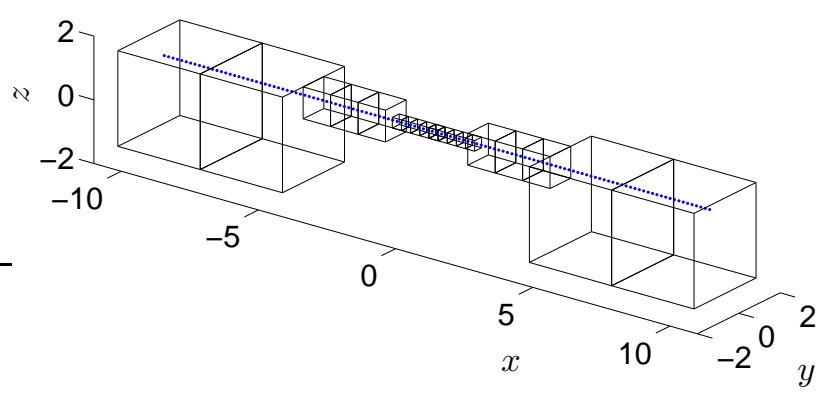

Fig. 2. The boxes that were generated in the storage tree for the bi-isotropic medium (79). The dotted line contains the sample points used to construct Figure 1.

with

$$
\begin{aligned}
\varepsilon_{t} & =2-0.01 j \\
\varepsilon_{z} & =1-0.01 j \\
\mu_{t} & =1.5-0.01 j, \\
\mu_{z} & =2.5-0.01 j .
\end{aligned}
$$

For a material of type (80), the Helmholtz determinant can be factorized as

$$
D(s)=\varepsilon_{t} \mu_{t}\left(\frac{\varepsilon_{z}}{\varepsilon_{t}} s_{z}^{2}+s_{x}^{2}+s_{y}^{2}-\varepsilon_{z} \mu_{t}\right)\left(\frac{\mu_{z}}{\mu_{t}} s_{z}^{2}+s_{x}^{2}+s_{y}^{2}-\mu_{z} \varepsilon_{t}\right) .
$$

The BSGF for such a Helmholtz determinant has been computed analytically in [18]. Figure 3 shows the comparison between the analytical BSGF and its numerically computed counterpart. The same derivatives are plotted as for the isotropic case. As can be seen, the agreement is very good and, even though this is not strictly enforced by the convergence criterium (71), the relative error between the analytical and numerical BSGF is everywhere smaller than the target accuracy $10^{-10}$.

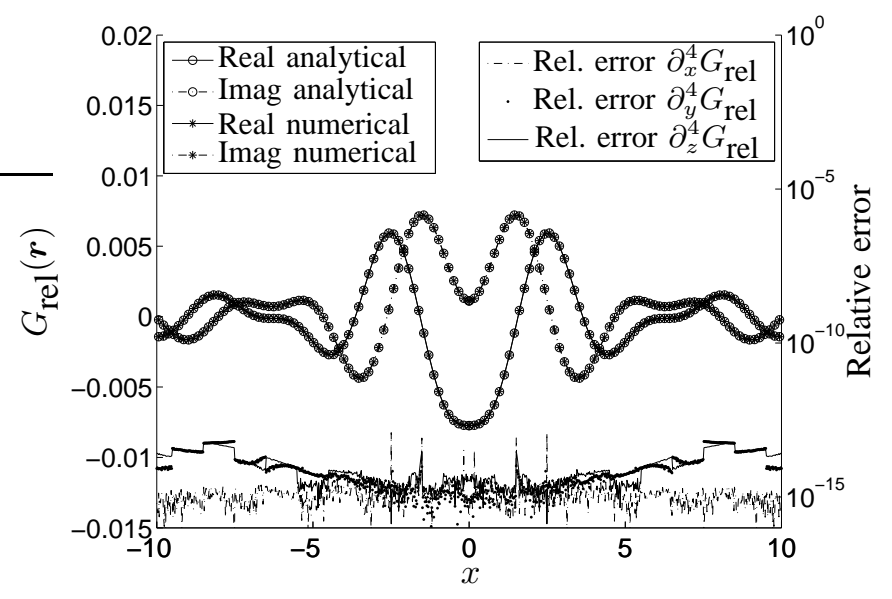

Fig. 3. A comparison between the numerically computed BSGF and its analytical counterpart for the uniaxial medium given in (80). Again, the right axis depicts the relative error between the numerical and analytical BSGF.

\section{A General Bianisotropic Material}

Finally, to show that our implementation also works for materials for which no analytical BSGF is known, a case with a completely general lossy bianisotropic material will be investigated. To avoid the pitfall of introducing too much symmetry into the material matrix, a random lossy bianisotropic material was generated. This was done by computing its relative material matrix $M$ as

$$
\mathrm{M}_{r}=\mathrm{C}_{1}+\mathrm{C}_{1}^{H}-j \alpha \sqrt{\mathrm{C}_{2}^{H} \cdot \mathrm{C}_{2}}
$$

where the complex matrices $C_{1}$ and $C_{2}$ have random elements, all with their real and imaginary part uniformly distributed in $[-1,1]$. The square root is taken of a symmetric positive definite matrix and is implemented by replacing the eigenvalues of the matrix by their positive square roots. If $\alpha=0$, the material is lossless [19]. If $\alpha$ is strictly positive, a lossy material is obtained. In this paper, $\alpha$ was chosen to be 0.01 , such that the BSGF does not decay too quickly. Finally, a number of materials were generated (86) with the aim of selecting one that does not oscillate too quickly in the coordinate range in Figure 4. This was done purely to limit the computation time, by limiting the number of boxes in the storage tree, since the very high accuracy that was set and the very complicated material can easily lead to long computation times. The material that was selected is given in Table I.

Figure 4 shows the BSGF and the error on it, computed as the inverse of the left hand side of (71). Note that the BSGF is not symmetric around the origin because the material used is not reciprocal nor isotropic. Clearly, criterium (71) is satisfied up to almost machine precision. This is implies that the derivatives (up to fourth order) are computed accurately.

\section{CONCLUSION}

A set of algorithms has been proposed for the computation and storage of the bianisotropic scalar Green's function for general lossy bianisotropic materials. This is a necessary first step towards the application of boundary integral equations for the modeling of electromagnetic scattering from bianisotropic 


\begin{tabular}{|l|l|l|}
\hline & Real part & Imag part \\
\hline \hline$\varepsilon_{x x, r}$ & 0.62370621668861004 & -0.04428010654312119 \\
\hline$\varepsilon_{y x, r}$ & 0.42195862981979115 & 1.17914056560005460 \\
\hline$\varepsilon_{z x, r}$ & 0.14519408757454633 & 0.45075308310701379 \\
\hline$\varepsilon_{x y, r}$ & 0.40327630295159300 & -1.18299549307799930 \\
\hline$\varepsilon_{y y, r}$ & 1.57295573927855910 & -0.03698156723376668 \\
\hline$\varepsilon_{z y, r}$ & 1.44752575374668520 & -1.29172509729574370 \\
\hline$\varepsilon_{x z, r}$ & 0.15246925663135524 & -0.43303970857205887 \\
\hline$\varepsilon_{y z, r}$ & 1.45162902856793160 & 1.29624868871419240 \\
\hline$\varepsilon_{z z, r}$ & -0.41210982890871817 & -0.04542391953919718 \\
\hline$\zeta_{x x, r}$ & -1.64014217473672710 & -0.88818413555297615 \\
\hline$\zeta_{y x, r}$ & 1.18467411089067820 & -1.22601314400278660 \\
\hline$\zeta_{z x, r}$ & 1.42990500871327340 & -1.21571205551113560 \\
\hline$\zeta_{x y, r}$ & -0.00570530361622299 & -0.70909945023886434 \\
\hline$\zeta_{y y, r}$ & -0.64856516626525751 & -0.37596480695969980 \\
\hline$\zeta_{z y, r}$ & 0.93087249985189269 & -1.43788960166005420 \\
\hline$\zeta_{x z, r}$ & -0.46453177657220479 & 0.39942172718798591 \\
\hline$\zeta_{y z, r}$ & 1.71147976577795750 & -0.04211260884344410 \\
\hline$\zeta_{z z, r}$ & 0.97894028319041393 & -0.98376795009122697 \\
\hline$\xi_{x x, r}$ & -1.63039748300194740 & 0.89803476096089396 \\
\hline$\xi_{y x, r}$ & -0.02098871303417807 & 0.70731681222139220 \\
\hline$\xi_{z x, r}$ & -0.45804633555586260 & -0.37311702338928948 \\
\hline$\xi_{x y, r}$ & 1.19170635170466180 & 1.23546261160499340 \\
\hline$\xi_{y y, r}$ & -0.64403236286703536 & 0.34429983833782518 \\
\hline$\xi_{z y, r}$ & 1.72696074272559060 & 0.02307564811232592 \\
\hline$\xi_{x z, r}$ & 1.41091703298848610 & 1.20106928517883600 \\
\hline$\xi_{y z, r}$ & 0.93411715835259912 & 1.43646839405541970 \\
\hline$\xi_{z z, r}$ & 0.98898545351365286 & 0.97486697845199088 \\
\hline$\mu_{x x, r}$ & -0.77613517791371844 & -0.03553636918305742 \\
\hline$\mu_{y x, r}$ & -0.82214566178004023 & 0.23037990549286638 \\
\hline$\mu_{z x, r}$ & 0.59586706559444158 & 0.77008039345416823 \\
\hline$\mu_{x y, r}$ & -0.82995494646103496 & -0.20728309697915678 \\
\hline$\mu_{y y, r}$ & -0.63220557727404314 & -0.04663963621353058 \\
\hline$\mu_{z y, r}$ & -1.17709932069501020 & 0.95134685284894749 \\
\hline$\mu_{x z, r}$ & 0.60548922245624626 & -0.76981537442914250 \\
\hline$\mu_{y z, r}$ & -1.16738338788591630 & -0.94108991269321951 \\
\hline$\mu_{z z, r}$ & -0.12783478686094396 & -0.02448853991714414 \\
\hline & & \\
\hline
\end{tabular}

TABLE I

THE RELATIVE MATERIAL PARAMETERS FOR THE USED RANDOM BIANISOTROPIC MATERIAL.

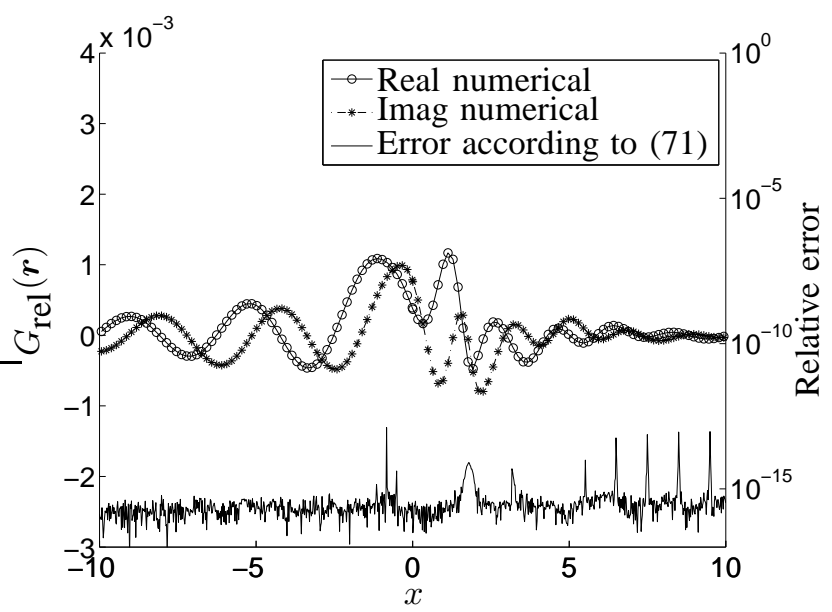

Fig. 4. The BSGF for the random bianisotropic material from Table I. The right axis is for the relative error, computed by means of (71). materials. The proposed algorithms use an expansion of the bianisotropic scalar Green's function into Chebyshev polynomials, which has great advantages from a numerical point of view. This Chebyshev expansion is coupled with a multilevel storage scheme that is error-controllable and adaptive. The proposed algorithms rely on a number of deep theoretical results, proved in this paper, that hold for the full set of lossy bianisotropic materials.

\section{APPENDIX A \\ Computation of $E, K$ And $S_{p}$}

In Section $\mathrm{V}$, the functions $E, K$ and $S_{p}$ were defined (see equations (58), (59) and (60) respectively). Here, entire versions of these functions will be defined and algorithms for their computation given. To define an entire version of a function of the type

$$
\mathcal{F}(x, y)=\frac{f(x)-f(y)}{x-y},
$$

it suffices to replace its undefined value on the removable singularity with $\frac{\mathrm{d}}{\mathrm{d} x} f(x)$. This can be done for all three functions that will be treated here. However, to compute them, some tricks are needed.

The function $E$ is the simplest case. It can be rewritten as

$$
E(x, y)=\frac{e^{j x}-e^{j y}}{x-y}=j \frac{\sin v}{v} e^{j s},
$$

with

$$
\begin{aligned}
& v=\frac{x-y}{2}, \\
& s=\frac{x+y}{2} .
\end{aligned}
$$

Expression (88), if evaluated numerically, maintains its accuracy even for very small $v$. If $v$ is exactly zero, then it should be evaluated as $j e^{j s}$. This allows $E(x, y)$ to be evaluated without catastrophic loss of accuracy in floating point arithmetic.

The computation of $K$ can be based on the computation of $E$, by means of

$$
K(x, y)=\frac{x e^{j x}-y e^{j y}}{x-y}=\frac{1}{2}\left[(x+y) E(x, y)+e^{j x}+e^{j y}\right] .
$$

The computation of $S_{p}$ is more complicated due to the presence of Bessel functions. However, it is still possible to robustly evaluate the entire version of this function. Starting from formula (10.23.2) in [7]

$$
J_{k}(a+b)=\sum_{n=-\infty}^{\infty} J_{k-n}(a) J_{n}(b), \forall a, b \in \mathbb{C}
$$

it is easily proved that

$$
\begin{aligned}
& J_{k}(x)=\sum_{n=-\infty}^{\infty} J_{k-n}(s) J_{n}(v), \\
& J_{k}(y)=\sum_{n=-\infty}^{\infty} J_{k-n}(s) J_{n}(-v) .
\end{aligned}
$$


Therefore:

$$
\begin{aligned}
S_{p}(x, y)= & \sum_{n=-\infty}^{\infty} J_{k-n}(s) \frac{J_{n}(v)}{x-y}\left[1-(-1)^{n}\right] \\
= & \sum_{n=-\infty}^{\infty} J_{k-2 n-1}(s) \frac{J_{2 n+2}(v)+J_{2 n}(v)}{4 n+2}, \\
= & \frac{1}{2} J_{0}(v)\left[J_{k-1}(s)-J_{k+1}(s)\right]+ \\
& \sum_{n=1}^{\infty} J_{2 n}(v)\left[\frac{J_{k-2 n+1}(s)-J_{k+2 n-1}(s)}{4 n-2}\right. \\
& \left.+\frac{J_{k-2 n-1}(s)-J_{k+2 n+1}(s)}{4 n+2}\right] .
\end{aligned}
$$

This expression is free from any removable singularities, but requires us to find a suitable $n$ to truncate the series. To do this, the use of the above expression will be limited to cases in which $|v|<\alpha$ with $\alpha$ a predetermined constant. If this inequality is not satisfied, then the direct evaluation of (60) is used. If $|v|<\alpha$, an a priori known upper bound $\mathcal{J}_{n}$ can be tabulated for the Bessel function $J_{2 n}(v)$ :

$$
\mathcal{J}_{n}=\max _{\phi \in[0,2 \pi]}\left|J_{2 n}\left(\alpha e^{j \phi}\right)\right| .
$$

However, one should be careful not to judge the convergence of series (97) based on this factor alone, since the factor between square brackets can in fact increase with rising $n$. In practice, this increase only occurs as long as $2 n \leq k$, such that the series can be truncated at $n=N$ with $N$ constrained from below by

$$
\frac{\mathcal{J}_{N}}{\mathcal{J}_{\left\lceil\frac{k}{2}\right\rceil}}<\text { Target accuracy. }
$$

\section{ACKNOWLEDGMENT}

The work of I. Bogaert was supported by a postdoctoral grant from the Fund for Scientific Research (Fonds Wetenschappelijk Onderzoek).

\section{REFERENCES}

[1] G. Mumcu, K. Sertel, and J. Volakis, "Surface integral equation solutions for modeling 3-d uniaxial media using closed-form dyadic green's functions," Antennas and Propagation, IEEE Transactions on, vol. 56, no. 8, pp. $2381-2388$, aug. 2008.

[2] S. Yarga, G. Mumcu, K. Sertel, and J. Volakis, "FEM and SIE modeling of layered uniaxial crystals for compact directive antennas," in Computational Electromagnetics Workshop, 2007, aug. 2007, pp. $70-74$.

[3] F. Olyslager and I. V. Lindell, "A pedigree of bianisotropic media." in Proceedings of the 8th International Conference on Electromagnetics of Complex Media (Bianisotropics 2000), 27-29 September 2000, Lisbon, Portugal, 2000, pp. 153-158.

[4] F. Olyslager and I. Lindell, "Electromagnetics and exotic media: a quest for the holy grail," Antennas and Propagation Magazine, IEEE, vol. 44, no. 2, pp. $48-58$, apr 2002.

[5] I. Lindell and F. Olyslager, "Generalized decomposition of electromagnetic fields in bi-anisotropic media," Antennas and Propagation, IEEE Transactions on, vol. 46, no. 10, pp. $1584-1585$, oct 1998.

[6] R. Harrington, Field Computations by Moment Methods. New York: Macmillan, 1968.

[7] F. W. J. Olver, D. W. Lozier, R. F. Boisvert, and C. C. W., NIST Handbook of Mathematical Functions. Cambridge University Press, 2010 .
[8] I. Bogaert, "Accurate Computation and Tabulation of the Scalar Green Function for Bi-anisotropic Media and its Derivatives," in Proceedings of the conference of the Applied Computational Electromagnetics Society, Columbus, Ohio, USA, April 2012.

[9] R.-C. Li, "Near optimality of chebyshev interpolation for elementary function computations," Computers, IEEE Transactions on, vol. 53, no. 6, pp. $678-687$, june 2004 .

[10] D. E. Amos, "Algorithm 644: A portable package for bessel functions of a complex argument and nonnegative order," ACM Trans. Math. Softw., vol. 12, no. 3, pp. 265-273, Sep. 1986. [Online]. Available: http://doi.acm.org/10.1145/7921.214331

[11] B. Jakoby and F. Olyslager, "Asymptotic expansions for greens dyadics in bianisotropic media," Progress on Electromagnetics Research, vol. 12, pp. 277-302, 1996.

[12] F. Olyslager and I. Lindell, "Field decomposition and factorization of the helmholtz determinant operator for bianisotropic media," Antennas and Propagation, IEEE Transactions on, vol. 49, no. 4, pp. $660-665$, apr 2001.

[13] G. Strang, Linear Algebra and its Applications, 3rd ed. Harcourt Brace Jovanovich, 1988.

[14] Branko Ćurgus and Vania Mascioni, "Roots and polynomials as homeomorphic spaces," Expositiones Mathematicae, vol. 24, no. 1, pp. 81-95, 2006. [Online]. Available: http://www.sciencedirect.com/science/article/pii/S0723086905000629

[15] Q. I. Rahman and G. Schmeisser, Analytic Theory of Polynomials. Oxford: Oxford University Press, 2002.

[16] L. V. Alhfors, Complex Analysis: an Introduction to the Theory of Analytic Functions of One Complex Variable, 2nd edition. New York: McGraw-Hill, 1966.

[17] N. J. Higham, "The accuracy of floating point summation," SIAM J. Sci. Comput, vol. 14, pp. 783-799, 1993.

[18] I. Lindell and F. Olyslager, "Polynomial operators and green functions abstract," Journal of Electromagnetic Waves and Applications, vol. 14, no. 8, pp. 1141-1142, 2000.

[19] I. V. Lindell and F. M. Dahl, "Conditions for the parameter dyadics of lossy bianisotropic media," Microwave and Optical Technology Letters, vol. 29, no. 3, pp. 175-178, May 2001.

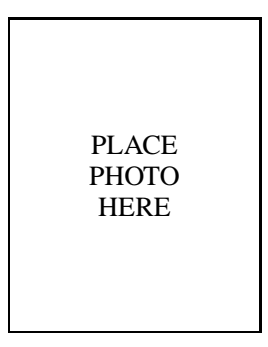

Ignace Bogaert Biography text here. 